\title{
SIMPLE ESTIMATION OF SURFACE ROUGHNESS PARAMETERS FROM SINGLE LEVEL SONIC ANEMOMETER DATA
}

\author{
Paolo Martano \\ CNR-ISIAtA, via Monteroni, 73100 Lecce, Italy. \\ Phone: +39 0832 320718; Fax: +39 0832320716 \\ p.martano@isiata.le.cnr.it
}

\begin{abstract}
The estimation of joint values of both the roughness length $z_{0}$ and the displacement height $d$ is considered in the context of the MoninObukhov similarity law for the windspeed profile. When focused on singlelevel data sets from one sonic anemometer (i.e. wind velocity, Reynolds stress and sensible heat flux data sets at one height), it is shown that this problem can be reduced to a simpler least squares procedure for one variable only. This procedure is carried out over a proper function of the data, representing the relative uncertainty of the roughness length, $\sigma_{z 0} / z_{0}$. This is minimized with respect to $d$, giving a direct estimate of $d, z_{0}$, and their statistical uncertainty. The scheme is tested against a field-experiment data set.
\end{abstract}




\section{INTRODUCTION}

It has been established that the mixing-length approximation, in the framework of similarity considerations describes the wind speed profile in the atmospheric surface layer in terms of the surface turbulent momentum and sensible heat fluxes.

In the equilibrium case the windspeed vertical profile appears to be governed by only one velocity and few height scales. In the MoninObukhov wind profile model scheme (Panofsky and Dutton, 1984), the velocity scale (friction velocity $u^{*}$ ) is directly related to the surface momentum flux, while one length scale (Obukhov length $L$ ) is related to both heat and momentum fluxes.

An other length scale appears as a boundary condition due to the presence of 'roughness elements' on the ground surface. This causes a local equilibrium breakdown by momentum transfer through the local pressure gradients at a height comparable with the obstacles vertical dimension, thus determining a new boundary scale $z_{0}$ (roughness length). When this is the case, another operational problem can take place. An uncertainty arises about the 'zero reference level' from which to measure the height $z$ above ground in the wind profile. If, anyway, $z$ is measured from the 'true' surface it would need the introduction of a 'displacement height' $d$. For example, in a flow over a forest, $d$ is related to the height of the trees, below which a wind profile in the previous sense does not hold. In this case, $z_{0}$ is related to the foliage shape and dimensions. From an operational point of view, and also in principle, the definition of those two heights is intrinsically related to the shape of the wind profile, so that their proper determination appears to be a fitting problem between flow data and theoretical similarity expressions. In the Monin-Obukhov similarity law, the windspeed profile $U(z)$ is written as:

$$
U(z)=(u * / k)\left[\ln \left((z-d) / z_{0}\right)-\psi\left((z-d) / L, z_{0} / L\right)\right]
$$

184 Rev. Ciência e Natura, Dispersion Process: 183 - 2000. 
where $u^{*}$ is the friction velocity scale, $L$ the Obukhov length, $k$ the von Karman constant, and $\psi((z-d) / L, z 0 / L)=\psi[(z-d) / L]-\psi(z 0 / L)$ is the integrated stability correction function (Panofsky and Dutton, 1984).

When applied to experimental data, this expression contains three kinds of parameters:

i) meteorological time averages, stochastically time dependent $\left(U, u^{*}\right.$ and $\left.L\right)$

ii) surface variables, depending on the involved site and area ( $z_{0}$ and $d$ )

iii) fixed parameters (the Von Karman constant and other non-dimensional parameters in the explicit expressions of $\psi$ ), considered as universal constants in the theory.

In the last decade the use of sonic anemometers in surfacelayer investigations has increased. When a sonic anemometer is used for single level measurements, the vertical kinematic fluxes $\langle w \theta\rangle$ and $\langle u w\rangle$ can be available along with time-averaged wind speed $U$ and temperature $T$ at one height $z$ (Cassardo et al. 1995), so that all the atmospheric variables in the Monin-Obukhov similarity law for wind speed are measured at the same time. (For the kinematic turbulent fluxes, the brackets <.. > indicate the usual time average and the small letters the turbulent fluctuations of horizontal $(u)$ and vertical (w) velocity and potential temperature $\theta$ ). Sozzi et al. (1998) used an iterative multivariate fitting procedure to estimate $z_{0}$ for different direction sectors and the non-dimensional parameters in the function $\psi$ from single level sonic anemometer data. They noted that the convergence of the fitting procedure is immediate (two steps) for the parameter zo. As will become clear in the following paragraph, this is not accidental: actually $z_{0}$ and the parameters contained in $\psi$ can be considered independently in the fitting procedure.

Focusing on the evaluation of $z_{0}$ and $d$, in the scheme of the Monin-Obukhov theory, and choosing the least squares method as best fit estimator, a mathematical formulation of the problem is: 


$$
<\left[k U / u^{*}-\ln \left((z-d) / z_{0}\right)+\psi\left((z-d) / L, z_{0} / L\right)\right]^{2}>_{m}=\min \left(z_{0}, d\right) .
$$

where the operator $\left\langle. .>_{m} \equiv(1 / N) \sum_{i}^{N}\right.$ is intended to be the average over the data set of $\mathrm{N}$ groups of time-averaged quantities $U_{i}, T_{i}, U_{*_{i}}, L_{i}$, at the same height z. Also: $u^{*}=(-<u w>)^{1 / 2}, \theta^{*}=-<w \theta>/ u^{*}, L=u^{2} T /\left(k g \theta^{*}\right)$, where $T$ is the absolute temperature, $g$ the gravitational acceleration $k$ the von Karman constant, and $\psi\left((z-d) / L, z_{0} / L\right)=\psi[(z-d) / L]-\psi\left(z_{0} / L\right)$ is the integrated stability correction function (Panofsky and Dutton, 1984).

The expression $\min \left(z_{0}, d\right)$ indicates the minimum with respect to both $z_{0}$ and $d$, and this is in principle a bidimensional non-linear least square problem. It will be shown in next section that it can be reduced to one-variable least square problem, that allows a simple estimate of $z_{0}$ and $d$ and their statistical uncertainties.

\section{MATHEMATICAL APPROACH}

Equation (1.2) can be written as:

$$
<\left[S\left(z_{0}, d\right)-p\left(z_{0}, d\right)\right]^{2}>=\min \left(z_{0}, d\right)
$$

where $S=\left[k U / u^{*}+\psi((z-d) / L)-\psi\left(z_{0} / L\right)\right]$ is a statistical quantity (function of the data) and $p=\ln \left((z-d) / z_{0}\right)$ is a parameter (function of $z, z_{0}$ and $d$ only). The index $\mathrm{m}$ has been dropped from the data-average brackets to simplify the notation: the operator $<. .>$ will coincide with $<. . ._{m}$ in the remaining part of the paper.

Consider now the related problem:

$$
<\left[S\left(z_{0}, d\right)-p\left(z_{0}, d\right)-<S\left(z_{0}, d\right)-p\left(z_{0}, d\right)>\right]^{2}>=\min \left(z_{0}, d\right) .
$$

The fact that $p$ is a parameter of the fitting procedure and not a measured quantity immediately implies that it is constant over the data set. Then $<p>=p$, so that:

$<\left[S\left(z_{0}, d\right)-p\left(z_{0}, d\right)-\left\langle S\left(z_{0}, d\right)-p\left(z_{0}, d\right)>\right]^{2}\right\rangle=\left\langle\left[S\left(z_{0}, d\right)-\left\langle S\left(z_{0}, d\right)>\right]^{2}\right\rangle=\sigma s^{2}\right.$

186 Rev. Ciência e Natura, Dispersion Process: 183 - 2000. 
Note now that (2.2) is equivalent to (1.2) with the condition

$$
<S\left(z_{0}, d\right)>-p\left(z_{0}, d\right)=0 \text { or } \ln \left((z-d) / z_{0}\right)=<k U / u^{*}+\psi\left((z-d) / L, z_{0} / L\right)>
$$

so that the problem of finding the minimum variance of $S=k U / u^{*}+\psi((z-$ $\left.d) / L, Z_{0} / L\right)$ with respect to $d$, with the constraint $\langle S\rangle=\ln \left((z-d) / z_{0}\right)$, appears to be equivalent to (1.2) which defines $z_{0}$ and $d$, and which has been reduced to a one-variable conditioned minimum problem.

Note, incidentally, that this is a general observation and is particularly useful when, as in the present case, a function of the parameters only $(p)$ is linear in the expression for the minimum, allowing an explicit solution for $p$ of the equation for the average value (here in the form $p=\langle S\rangle$ ).

Furthermore, it has been established that $\psi\left(z_{0} / L\right)=O\left(z_{0} / L\right)$ if $/ z_{0} / L /<<1$, as the limit condition for neutral flow $\psi(0)=0$ must hold. This means that in the usual measurement conditions, when $/ z_{0} / L /<</(z-d) / L /<<1, \quad / \psi((z-$ $d) / L />>/ \psi\left(z_{0} / L\right) /$ and $\psi\left[(z-d) / L, z_{0} / L\right]=\psi[(z-d) / L]-\psi\left(z_{0} / L\right) \cong \psi[(z-d) / L]$ (this is indeed the usual form of the stability correction in the surface layer, see e.g. Garratt, 1992; Panofsky and Dutton, 1984). This means that $S$ will be independent of $z o$, and the conditioned minimum problem for $\sigma s^{2}\left(d, z_{0}\right)$ becomes a simple one-dimensional minimum problem for $\sigma s^{2}(d, 0)$ in which the 'constraint' $\langle S\rangle=\ln \left((z-d) / z_{0}\right)$ will only be used to find $z_{0}$, after the value of $d$ that minimizes $\sigma s^{2}$ has been found.

The quantity $\sigma s^{2}$ to be minimized with respect to $d$ can attain a more intuitive meaning noting that the constraint (2.4) would be the best estimate of $z$. in the least square sense if $d$ were known. If we estimate the standard

deviation of $z_{0}$ by taking $z_{0}=z_{0 e}+\Delta z_{0}$ and using the 'single point' relationship $z_{0}$ $=(z-d) \exp (-S)$, and expanding in Taylor series up to first order in $S=\langle S\rangle+\Delta S$,, taking into account (2.4) we find: 
$\sigma_{z 0}=(z-d) \exp <-S>\sigma S$

so that

$$
\sigma \mathrm{s} \approx \sigma_{\mathrm{zo}} / \mathrm{Z}_{0 \mathrm{e}} .
$$

An estimate of the statistical uncertainty over $d$ can also be determined, noting that it coincides with that of $(z-d)$, which again can be estimated by means of a Taylor expansion, after having highlighted $(z-d)$ from the logarithm in (2.4). After a straightforward calculation, the result is:

$$
\sigma_{\mathrm{d}} \approx(z-d) \sigma \mathrm{s}
$$

which shows that the uncertainty in $d$ increases with the measurement height, as expected.

It can be noted that the minimization of $\sigma$ s can be carried out with respect to any other parameter in place of $d$, (for example a non-dimensional constant of the function $\psi$ ), maintaining the relative independence from $z_{0}$. This could explain the fast convergence of the iterative equation for $z_{0}$ in the fitting procedure by Sozzi et al. (1998).

Eventually, an even more practical approximation can be used noting that, if $\sigma s$ is sufficiently small, the statistical averaging and exponential operators over $S$ can be exchanged, as can be seen by using a second order Taylor expansion around <-S> for the exponential function:

$<\exp (-S)>\cong \exp <-S>\left[1+(1 / 2) \sigma s^{2}\right]$

If $\sigma s^{2}<<1$ (we are attempting to establish the minimum of $\sigma s$ : see fig. 1 for a typical value), it follows at once from (1.3):

$$
z_{0 e}=(z-d) \exp <-S>\cong(z-d)<\exp (-S)>=\left\langle z_{0}\right\rangle_{m}
$$

This suggests that a practical approximation for $d, z_{0}$ and their uncertainty can be obtained by calculating the ratio between the standard deviation of $z o$ and 
its average value over the whole data set, and finding its minimum with respect to $d$.

\section{APPLICATION TO FIELD DATA}

In this example of application three practical procedures are compared to estimate the best values of $d$ and $z_{0}$, based on the results of section 2.

1) The exact procedure of minimizing $\sigma s\left(d, z_{0}\right)$ with respect to $d$ under the constraint that $z_{0}(d)=(z-d) \exp <-S>$. This implies a calculation of $\sigma s$ changing $d$ step-by-step from slightly negative values up to the maximum allowed $d=z$, while using in $\sigma s\left(d, z_{0}\right)$ the value of $z_{0}$ calculated iteratively at each step by Eq.(2.4). This is equivalent to solving the exact least square problem of Eq. (1.2) with respect to both $z_{0}$ and $d$, as discussed, and it has also been proven numerically.

2) Minimizing os step-by-step with respect to $d$ as above, but now with the simpler procedure of using the approximation $\psi\left((z-d) / L, z_{0} / L\right) \cong \psi((z-d) / L, 0)=$ $\psi[(z-d) / L]$, so that $\sigma s^{2}=\sigma s^{2}(d, 0)$ is independent on $z_{0}$ (unconditioned minimum, one does not need to calculate $z_{0}$ at each step).

In these two cases, the final value of $z_{0}$ is given by (2.4), with $d$ corresponding to the minimum of $\sigma s$.

3) The approximate procedure of finding the minimum of $\sigma_{z 0} / z_{0}$ e with respect to $d$, using the same approximation as in point 2), which gives directly the values of $z_{0 e}, \sigma_{z 0}$ and $d$.

The data set consists of simultaneous 30 minute averages of wind speed, sensible heat flux, momentum flux and temperature, taken at the top of a mast of $z=12 \mathrm{~m}$ height by a Solent tri-axial sonic anemometer. Flux data were were obtained directly by eddy-correlation in real time through 
home-made software that also provided a rotation in the 'streamline' reference frame (McMillen,1988) to eliminate the effect of vertical misalignment and to give the proper value of the Reynolds stress in the windspeed-oriented reference frame. The measurement site was on flat terrain at about $5 \mathrm{~km} \mathrm{~W}$ of the town of Lecce, Italy, about $10 \mathrm{~km}$ from the Adriatic coast and $20 \mathrm{~km}$ from the lonic coast of the Sallentum peninsula. The mast was placed in a small clearing (less than $100 \mathrm{~m}$ of radius) on the university campus. The area surrounding the clearing is covered by trees with a height between 5 and $10 \mathrm{~m}$ (mainly pine, eucalyptus, cypress and some olive trees), at a minimum distance of about $50 \mathrm{~m}$ from the mast (short olive trees and cypresses), up to more then $1 \mathrm{~km}$ in all directions. Some isolated two-storied buildings are also present in the area around the clearing.

An example of the obtained results is shown in Fig 1, where the above three procedures have been applied to a single wind direction sector of $30^{\circ}$ of width in the data set, to reduce the scatter (wind speeds less than $1.5 \mathrm{~m} \mathrm{~s}^{-1}$ were also dropped from the data set).

The Businger-Dyer forms for the $\psi$ functions have always been used (see e.g. Panofsky and Dutton, 1984) and a $0.5 \mathrm{~m}$ step was always used in the minimum procedure with respect to $d$, that is less than its statistical uncertainty (see below).

Procedure 1) gives $z_{0}=.37$ and $d=8 \mathrm{~m}$, and fig 1 shows that the results for procedures 2 ) and 3) lie within the statistical uncertainty $\sigma_{z 0}=.10$ and $\sigma_{d}=$ $1.1 \mathrm{~m}$ (calculated from eqs. 2.5 and 2.6). These values can be reasonably

compared with those reported in the literature for similar terrain conditions (see e.g. Wieringa, 1993, for a very good review).

Figure 2 shows a test of consistency of the obtained results. Here the MoninObukhov similarity windspeeds are calculated with the values of $z_{0}$ and $d$ obtained by procedure 1), and compared with the measured ones. The 190 Rev. Ciência e Natura, Dispersion Process: 183 - 194 ,2000. 
straight lines are minimum chi-square $\left(\chi^{2}\right)$ bestfit lines, that enable the reliability of the used model parameters $\left(z_{0}\right.$ and $\left.d\right)$ to be tested, where:

$$
\chi^{2}=[1 /(N-P)] \Sigma^{N}\left(U_{E i}-U_{M i}\right)^{2} / \sigma^{2}
$$

$U_{M i}$ are the measured windspeeds, $U_{E i}=\left(u^{*} / k\right)\left(S-\psi_{i}\right)$ the windspeeds estimated from (1.1), $\mathrm{N}$ the number of data, and $\mathrm{P}=2$ the number of parameters estimated from the data set by the model equations ( $z_{0}$ and $d$ ). To test the consistency of the estimates of $z_{0}$ and $d$, the measured data are considered as reference values and the statistical uncertainty $\sigma_{i}$ of each estimated windspeed $U_{E i}$ is considered as dependent on the uncertainty of the roughness parameters only, and calculated as follows:

$\sigma^{2}=\left(u^{*} / k\right)^{2}\left[\sigma^{2}-2 \operatorname{cov}\left(S, \psi_{i}\right)+\sigma_{\psi i}^{2}\right]=\left(u^{*} / k\right)^{2} \sigma s^{2}[1+O([z-d] / L i)] \approx\left(u^{*} / k\right)^{2} \sigma S^{2}$

where $\sigma_{\psi i t}{ }^{2}$ is the variance (function of $\sigma_{d}$ ) of the function $\psi_{i}$ and $\operatorname{cov}\left(\mathrm{S}, \psi_{i}\right)$ indicates the covariance between $S=\ln \left((z-d) / z_{0}\right)$ and $\psi_{i}=\psi\left([z-d] / L_{i}\right)$.

The last two equalities hold for $/(z-d) / L /<<1$ (a condition verified in the used data sets), in which case it can be inferred from the results of section 2 that $\operatorname{cov}\left(S, \psi_{i}\right) / \sigma s^{2}=O\left([z-d] / L_{i}\right)$ and $\sigma \psi_{i j}^{2} / \sigma s^{2}=O\left(\left[(z-d) / L_{i}\right]^{2}\right)$.

The regression coefficient is $r=0.94 \quad(\mathrm{~N}=100$ data). For the minimum chisquare of (3.1), whose expected value is 1 , it was obtained: $\chi^{2}=1.27$ which corresponds to a confidence coefficient of 0.95 in the $\chi^{2}$ distribution for $\mathrm{N}$ $\mathrm{P}=98$.

\section{CONCLUDING REMARKS}

Surface roughness and displacement height can be determined from the similarity wind profile law from single level measurements of windspeed and fluxes by solving a simple and straightforward onedimensional minimum problem. The quantity to be minimized has a direct physical meaning and allows the estimation of the statistical uncertainties of the two parameters. The procedure requires no specialized software, very 
little computational effort and can also be used to estimate different parameters in the stability correction functions.

\section{REFERENCES}

Cassardo C., D. Sacchetti , M.G. Morselli, D. Anfossi, G. Brusasca and A. Longhetto, 1995: A study of the assessment of air temperature and sensible-heat and latent-heat fluxes from sonic anemometer observations. II Nuovo Cimento C, 4, 419-440.

Garratt J.R.,1992: The Atmospheric Boundary Layer. Cambridge University Press, $316 \mathrm{pp}$.

McMillen R.T.,1988: An eddy correlation tecnique with extended applicability to non-simple terrain. Bound.-Layer Meteor., 43, 231-245.

Panofsky H.A.and J.A. Dutton, 1984: Atmospheric Turbulence. John Wiley \& Sons, New York, 397 pp.

Sozzi R., M. Favaron, T.Georgiadis, 1998: Method for the estimation of surface roughness and similarity function of wind speed vertical profile. J. Appl. Meteor., 37, 461-469.

Wieringa J.,1993: Representative roughness parameters for homogeneous terrain. Bound.-Layer Meteor. 63, 323-363.

$\sigma s$

192 Rev. Ciência e Natura, Dispersion Process: 183 - 194 ,2000. 


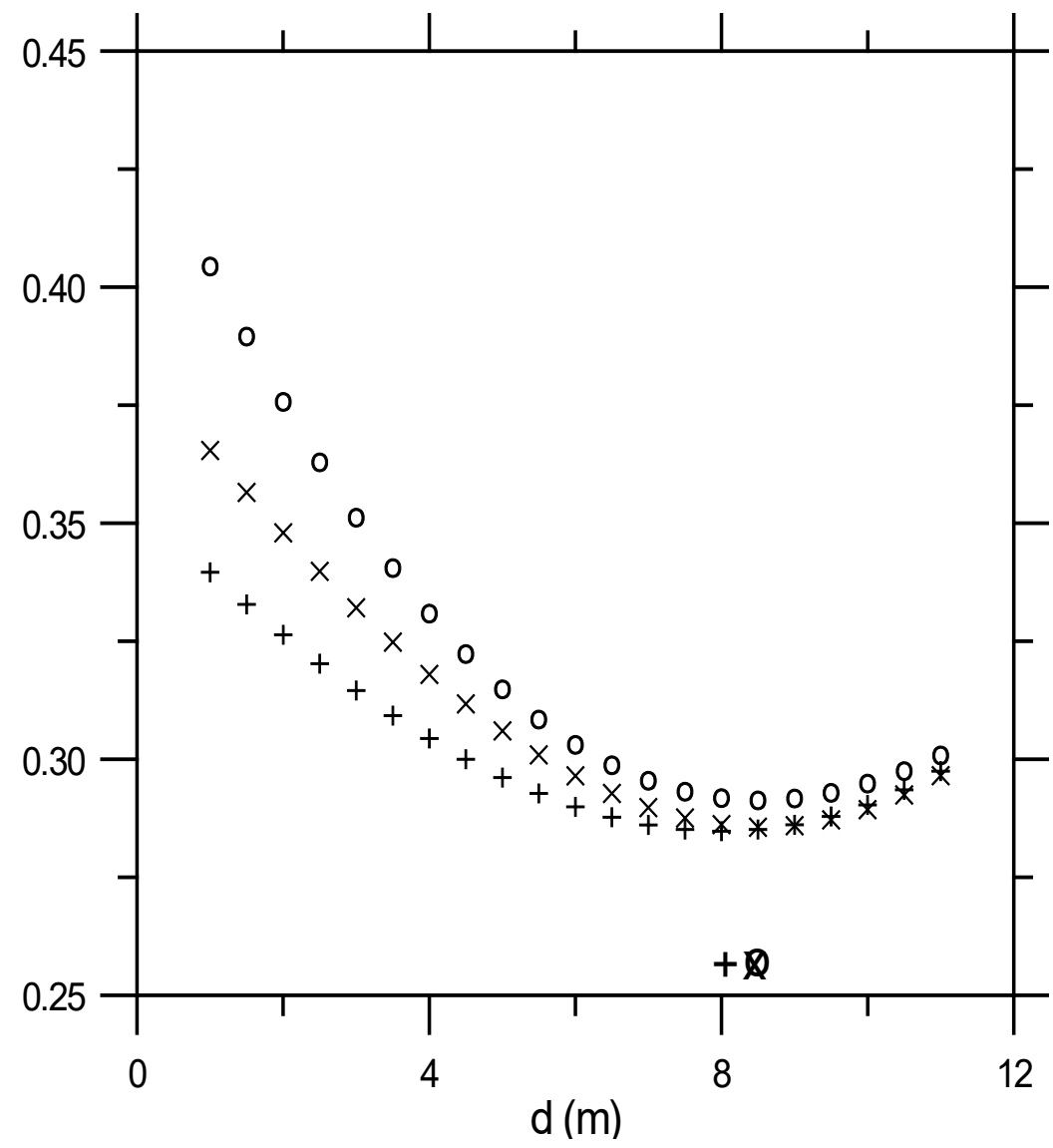

FIGURE 1: Plot of the standard deviation $\sigma s$ of $S=k U / u^{*}+\psi((z-d) / L, Z 0 / L)$ versus $d$ for a $30^{\circ}$ width sector in the data set:

- as function of $d$ and $z_{0}: \sigma s\left(d, z_{0}\right)$ (plus signs),

- as function of $d$ only: $\sigma s(d, 0)$ (crosses),

- for the approximation $\sigma_{S} \approx \sigma_{z} 0 /<z_{0}>$ (circles).

The symbols close to the $d$ axis show the position of the minima of the corresponding curve, and the predicted values of $d$.

$U_{E}$

Rev. Ciência e Natura, Dispersion Process: 183 - 2000. 


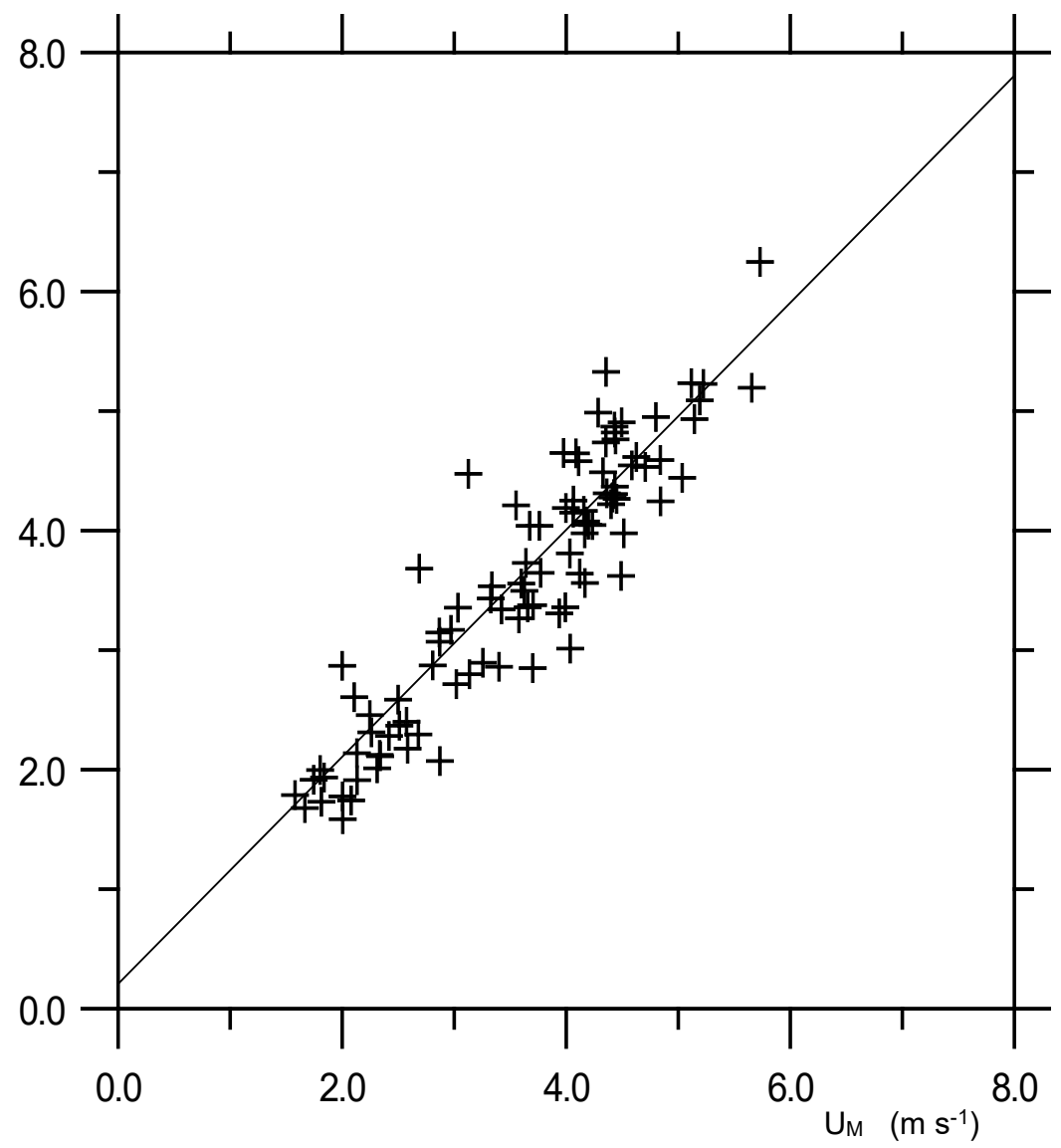

FIGURE 2: Plot of the wind speeds estimated by the Businger-Dyer forms of the Monin-Obukhov similarity $U_{E}$ versus the measured wind speeds $U_{M}$. The data set and the used estimates of $d$ and $z_{0}$ are those of Fig 1. The straight line represents a least chi-square best fit.

194 Rev. Ciência e Natura, Dispersion Process: 183 - 194 ,2000. 